\title{
Balanced Subdivision and Enumeration in Balanced Spheres
}

\author{
Louis J. Billera ${ }^{1 *}$ and Katherine E. Magurn ${ }^{2 *}$ \\ 'Department of Mathematics and School of Operations Research, Cornell University, \\ Ithaca, NY 14853, USA, and Department of Mathematics and Center for Operations Research, \\ Rutgers University, New Brunswick, NJ 08903, USA \\ ${ }^{2}$ Department of Mathematics and Statistics, Miami University, Oxford, OH 45056, USA
}

\begin{abstract}
We study here the affine space generated by the extended $f$-vectors of simplicial homology $(d-1)$-spheres which are balanced of a given type. This space is determined, and its dimension is computed, by deriving a balanced version of the Dehn-Sommerville equations and exhibiting a set of balanced polytopes whose extended $f$-vectors span it. To this end, a unique minimal complex of a given type is defined, along with a balanced version of stellar subdivision, and such a subdivision of a face in a minimal complex is realized as the boundary complex of a balanced polytope. For these complexes, we obtain an explicit computation of their extended $h$-vectors, and, implicitly, $f$-vectors.
\end{abstract}

\section{Introduction}

In general terms, this paper investigates balanced simplicial complexes. These are complexes whose vertices are labeled by $1,2, \ldots, m$ in such a way that each maximal face has $a_{i}$ vertices with label $i, 1 \leq i \leq m$. Such complexes are said to be balanced of type $a$, where $a=\left(a_{1}, \ldots, a_{m}\right)$. In particular, we generalize to the balanced case some results of [3], [4], and [5] for completely balanced complexes, i.e., balanced complexes of type $(1, \ldots, 1)$. For a balanced simplicial complex $\Delta$, let $f_{b}(\Delta)$ denote the number of faces of $\Delta$ having $b_{i}$ vertices with label $i$, where $b=\left(b_{1}, \ldots, b_{m}\right)$. The vector $f(\Delta)=\left(f_{b}(\Delta)\right)_{b \leq a}$ is called the extended f-vector of $\Delta$. We determine here the affine space generated by the extended $f$-vectors of simplicial homology $(d-1)$-spheres which are balanced of a given type, thus determining all possible affine relations satisfied by their components.

\footnotetext{
* Supported in part by NSF Grant DMS-8403225.
} 
We now present the definitions and basic facts to be used throughout the paper. Let $\sigma \in \Delta$, a simplicial complex. Then the link of $\sigma$ in $\Delta$ is defined to be $l k_{\Delta} \sigma=\{\tau \in \Delta: \sigma \cap \tau=\varnothing, \sigma \cup \tau \in \Delta\}$. If $\sigma \neq \varnothing$, the deletion of $\sigma$ in $\Delta$ is $\Delta \backslash \sigma \equiv$ $\{\tau \in \Delta: \sigma \not \subset \tau\}$. If $\Delta_{1}$ and $\Delta_{2}$ are simplicial complexes with no vertices in common, then their join is defined to be $\Delta_{1} * \Delta_{2}=\left\{\sigma_{1} \cup \sigma_{2}: \sigma_{1} \in \Delta_{1}, \sigma_{2} \in \Delta_{2}\right\}$. Note that each of these operations yields a simplicial complex. For $\sigma \in \Delta$ and $v$ a vertex not in $\Delta$, the stellar subdivision of $\sigma$ in $\Delta$ is the complex $\operatorname{st}_{\Delta}(\sigma, v) \equiv(\Delta \backslash \sigma) \cup \bar{v} * \partial \bar{\sigma} * l k_{\Delta} \sigma$. Here $\bar{\sigma}$ is the complex consisting of all subsets of $\sigma ; \bar{v} \equiv\{\overline{v\}}$.

Of historical interest is Alexander's "arithmetic" describing stellar subdivision on which a whole theory of stellar equivalence is based [1]. Whether such a theory can be developed for balanced complexes is a question of some interest, but beyond the scope of this paper (see [15].) Stellar subdivision may also be defined for polytopes. For a discussion in the most general (nonsimplicial) case, see $[9]$.

A $(d-1)$-dimensional simplicial complex is said to be a homology $(d-1)$ sphere if for each $k$-face $\sigma \in \Delta, l k_{\Delta} \sigma$ is a $(d-k-2)$-dimensional complex having the rational homology of a $(d-k-2)$-sphere, $-1 \leq k \leq d-1$. We denote by $C_{a}^{d}$ the set of all type $a$ balanced complexes that are homology $(d-1)$-spheres.

If $\Delta$ is a $(d-1)$-dimensional simplicial complex, then for $-1 \leq i \leq d-1$, we denote the number of faces of $\Delta$ having dimension $i$ by $f_{i}=f_{i}(\Delta)$. The $f$-vector and $h$-vector of $\Delta$ are defined by

$$
f=\left(f_{-1}, f_{0}, \ldots, f_{d-1}\right) \text { and } h=\left(h_{0}, h_{1}, \ldots, h_{d}\right)
$$

where

$$
h_{i}=\sum_{j=0}^{i}(-1)^{i-j}\left(\begin{array}{l}
d-j \\
d-i
\end{array}\right) f_{j-1} .
$$

We can define an extended $h$-vector $h(\Delta)=\left(h_{b}(\Delta)\right)_{b \leq a}$ of a balanced complex (of type $a$ ) by

$$
h_{b}(\Delta)=\sum_{c \leq b} f_{c}(\Delta) \prod_{i=1}^{m}(-1)^{b_{i}-c_{i}}\left(\begin{array}{l}
a_{i}-c_{i} \\
b_{i}-c_{i}
\end{array}\right) .
$$

An alternative definition, more useful for our purposes, is the following. See [21] for details. Let $t$ represent the multivariable $\left(t_{1}, \ldots, t_{m}\right)$, and if $b=\left(b_{1}, \ldots, b_{m}\right) \in$ $\mathbb{N}^{m}$, let $\mathbf{t}^{b}$ denote $t_{1}^{b_{1}} \cdots \cdots t_{m}^{b_{m}}$, and define the polynomial $f(\Delta, \mathbf{t}) \equiv \sum_{b \leq a} f_{b}(\Delta) \mathbf{t}^{b}$. For $w=\left(w_{1}, \ldots, w_{m}\right) \in \mathbb{N}^{m}$, define $h^{(w)}(\Delta, t) \equiv(1-t)^{w} f(\Delta, t / 1-t)$ where the notation $(1-t)^{w}$ means $\prod_{i=1}^{m}\left(1-t_{i}\right)^{w_{i}}$. When $w=a$, the balanced type of $\Delta$, we distinguish $h^{(a)}(\Delta, t)=(1-t)^{a} f(\Delta, t / 1-t)$ as $h(\Delta, t)$, defining the coefficient of $t^{b}$ in $h(\Delta, t)$ to be $h_{b}(\Delta)$. Both the extended $f$-vector and the extended $h$-vector are refinements of the usual $f$-vector and $h$-vector.

Two useful interpretations of the number $h_{b}(\Delta)$ have been supplied by Stanley. See [21] for details of both. First, $h_{b}(\Delta)$ is the vector space dimension of the $b$ th summand in the usual $\mathbb{N}^{m}$-grading on the Stanley-Reisner ring of $\Delta \bmod$ a homogeneous system of parameters. Secondly, if $\Delta$ is balanced of type $a$ and $\sigma_{1}, \ldots, \sigma_{s}$ is a shelling of $\Delta$, then $h_{b}(\Delta)$ counts the number of times it happens in the shelling process that the unique minimal face of $\sigma_{i}(1 \leq i \leq s)$ which is not contained in $\sigma_{1} \cup \cdots \cup \sigma_{i-1}$ is type $b$. 
The linear relation defining the (extended) $h$-vector from the (extended) $f$-vector is invertible, so a description of the affine span of all $h$-vectors will implicitly give a description of the span of all $f$-vectors.

It is the main result of this paper that the affine span of the extended $h$-vectors of balanced homology spheres (of type $a$ ) is given by the equations $h_{b}(\Delta)=$ $h_{a-b}(\Delta)$ for all $b \leq a$. This description reduces to that given by the classical Dehn-Sommerville equations for the usual $f$-vectors $\left(h_{i}=h_{d-i}, 0 \leq i \leq d\right.$, in the $h$-vector form) of simplicial homology spheres (in particular, simplicial convex polytopes) [11], [13], [19], [4], and to that obtained for the completely balanced case in [4] $\left(h_{S}=h_{\tilde{S}}\right.$ for $S \subseteq\{1, \ldots, d\}$, where $h_{S} \equiv h_{b}, b$ the characteristic vector of the set $S$ ). In the former case, $a=(d)$ (all vertices receive the same label) and in the latter, $a=(1,1, \ldots, 1)$.

\section{Symmetry of the Extended $h$-vector}

This section specifies certain linear equations which the coordinates of every extended $h$-vector satisfy. In addition to these, each $h$-vector satisfies the balanced version of Euler's relation, and this collection of equations will later be shown to characterize the space $\operatorname{aff}\left(h_{b}\left(C_{a}^{d}\right)\right)$. The symmetric nature of the extended $h$-vector is displayed in the following theorem, whose proof is a straightforward generalization of a proof of Bayer and Billera [4, p. 235], which in turn is based on one of Stanley's [22]. For details, see [15]. We remark that in the case of shellable spheres (e.g., polytopes), a purely combinatorial proof is possible, using Proposition 3.6 of [21]. See Proposition 5.1 of [4] for such a proof in the completely balanced case.

Theorem 1. Let $\Delta \in C_{a}^{d}, a=\left(a_{1}, \ldots, a_{m}\right)$. Then $h_{b}(\Delta)=h_{a-b}(\Delta)$ for all $b \leq a$.

This theorem is enough to determine an upper bound on the dimension of $\operatorname{aff}\left(h_{b}\left(C_{a}^{d}\right)\right)$. In what follows, let $K=\prod_{i=1}^{m}\left(a_{i}+1\right)$.

Corollary 2. $\operatorname{dim} \operatorname{aff}\left(h_{b}\left(C_{a}^{d}\right)\right) \leq\lfloor(K-1) / 2\rfloor$.

Proof. For all $\Delta \in C_{a}^{d}$ we have $h_{0}=1$ and $h_{b}(\Delta)=h_{a-b}(\Delta)$ for all $b \leq a$. These are independent linear equations and there are $\lfloor K / 2\rfloor+1$ of them, so they determine an affine subspace of dimension $K-(\lfloor K / 2\rfloor+1)=\lfloor(K-1) / 2\rfloor$. Finally, aff $\left(h_{b}\left(C_{a}^{d}\right)\right)$ is contained in this subspace, and so $\operatorname{dim} \operatorname{aff}\left(h_{b}\left(C_{a}^{d}\right)\right) \leq$ $\lfloor(K-1) / 2\rfloor$.

\section{The Minimal Type a Complex}

It is known that if $S$ is an unlabeled $d$-simplex, $h(\partial S)$ consists of $d+11$ 's followed by the appropriate number of 0's [4]. This section offers a description of certain polytopes whose boundary complexes, in the balanced case, act as analogues to $\partial S$. The extended $h$-vectors of these complexes are computed, and are shown to consist of 1 's. 
First, we describe a basic construction. Suppose $P_{i}$ is a $d_{i}$-polytope in $\mathbb{R}^{d_{i}}$ with $0 \in$ int $P_{i}, i=1,2$. Let $\bar{P}_{1}=P_{1} \times\{0\}$ and $\bar{P}_{2}=\{0\} \times P_{2}$ be embeddings of $P_{i}$ in $\mathbb{R}^{d}$, $d=d_{1}+d_{2}$, and define $P_{1} \# P_{2} \equiv \operatorname{conv}\left(\bar{P}_{1} \cup \bar{P}_{2}\right)$. In the case in which $P_{1}$ and $P_{2}$ are simplicial polytopes, it is straightforward to verify that

$$
\partial\left(P_{1} \# P_{2}\right)=\partial P_{1} * \partial P_{2} \text {. }
$$

Suppose $a=\left(a_{1}, \ldots, a_{m}\right)$ and let $d \equiv \sum_{i=1}^{m} a_{i}$. For $1 \leq i \leq m$, let $S_{i}$ be an $a_{i^{-}}$ simplex in $\mathbb{R}^{\alpha_{i}}$ with $\mathbf{O}$ in its interior, and with vertices labeled $i$. Assume $S_{1}, \ldots, S_{m}$ are embedded in $\mathbb{R}^{d}$, as above, and define $\hat{\Delta} \equiv \partial\left(S_{1} \# S_{2} \# \cdots \# S_{m}\right)=$ $\partial S_{1} * \partial S_{2} * \cdots * \partial S_{m}$. It follows that $\hat{\Delta}$ is type $a$, for a maximal simplex of $\partial S_{i}$ has exactly $a_{i}$ vertices. We call $\hat{\Delta}$ the minimal type a complex.

\section{Examples}

(1) $a=(2,1)$

$\hat{\Delta}=\partial S_{1} * \partial S_{2}$, where $S_{1}$ is a 2 -simplex labeled 1 , and $S_{2}$ is a 1 -simplex labeled 2 .

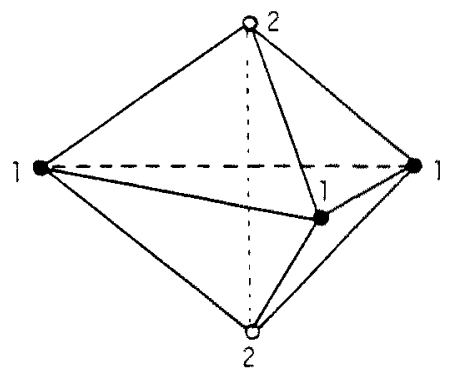

(2) $a=(1,1,1)$

$\hat{\Delta}=\partial S_{1} * \partial S_{2} * \partial S_{3}$, where, for $1 \leq i \leq 3, S_{i}$ is a 1 -simplex labeled $i$.

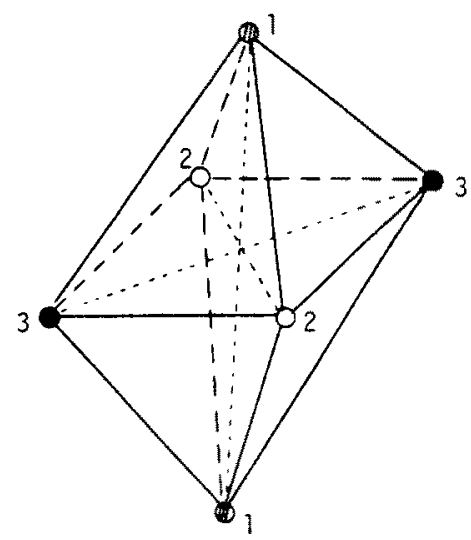

Remark 1. $\hat{\Delta}$ is minimal in the sense that it has the smallest number of vertices of any homology sphere of type $a$. For, if $\Delta$ is a homology sphere of type $a$, the number of vertices of $\Delta$ with label $i$ must be at least $a_{i}$, by definition of "type $a$ ". Suppose for some $i$ that the number of vertices with label $i$ is exactly $a_{i}$. 
Then each facet of $\Delta$ contains all the vertices with label $i$, that is, a vertex $v$ with label $i$ is contained in all the facets of $\Delta$. But then for any facet $F$ of $\Delta, F \backslash\{v\}$ is a subfacet included in a unique facet, which is impossible. Thus $\hat{\Delta}$ is minimal among type $a$ homology spheres in terms of number of vertices.

For a given type, how many vertices is this? If $a=\left(a_{1}, \ldots, a_{m}\right)$ and $a_{i} \neq 0$ for all $1 \leq i \leq m$, then clearly $f_{0}(\hat{\Delta})=\sum_{i=1}^{m}\left(a_{i}+1\right)$. An adjustment must be made if $a_{i}=0$ for some $i$. (This situation occurs in the next section.) In the construction of $\hat{\Delta}, a_{i}=0$ indicates a 0 -simplex $v$, labeled $i . v$ is placed on the origin in the embedding, so when the convex hull of all the simplices is taken to make $\hat{\Delta}, v$ disappears into the interior of the polytope. Thus $a_{i}=0$ contributes no vertices to $\hat{\Delta}$. So, in general,

$$
f_{0} \overline{(\hat{\Delta})}=\sum_{i=1}^{m}\left(a_{i}+1\right)-\left|\left\{i: a_{i}=0\right\}\right|
$$

Remark 2. It is also true that $\hat{\Delta}$ is unique among the homology spheres of type $a=\left(a_{1}, \ldots, a_{m}\right)$ having $a_{i}+1$ vertices labeled $i, 1 \leq i \leq m$. For if there exists another such complex $\Gamma$, it can be embedded as a full-dimensional subcomplex of $\hat{\Delta}$. Then since $\Gamma$ itself has 0 boundary, $\Gamma$ must be all of $\hat{\Delta}$. (Alternatively, one can use "invariance of domain." See [16] and [12].)

The rest of this section is devoted to the computation of $h_{b}(\hat{\Delta})$ for $b \leq a$.

Proposition 3. Let $\Delta_{1}$ and $\Delta_{2}$ be balanced complexes. Then $\Delta_{1} * \Delta_{2}$ is balanced, and $h_{b}\left(\Delta_{1} * \Delta_{2}\right)=\sum_{v+w=b} h_{v}\left(\Delta_{1}\right) \cdot h_{w}\left(\Delta_{2}\right)$.

Proof. Choose an order for the set of distinct labels appearing in $\Delta_{1}$ or $\Delta_{2}$, and write the vectors describing the type of each complex according to this order, using 0 's where necessary.

Suppose $\Delta_{1}$ is type $a=\left(a_{1}, \ldots, a_{m}\right)$ and $\Delta_{2}$ is type $a^{\prime}=\left(a_{1}^{\prime}, \ldots, a_{m}^{\prime}\right)$. A maximal simplex in $\Delta_{1} * \Delta_{2}$ is the union of a maximal simplex in $\Delta_{1}$ with a maximal simplex in $\Delta_{2}$, and it is clear that $\Delta_{1} * \Delta_{2}$ is balanced of type $a+a^{\prime}$.

In what follows, let $t$ denote the multivariable $\left(t_{1}, \ldots, t_{m}\right)$ and let $(1-t)^{v}$ denote the product $\left(1-t_{1}\right)^{v_{1}} \ldots \ldots\left(1-t_{m}\right)^{v_{m}}$ for $v=\left(v_{1}, \ldots, v_{m}\right)$. Since $f_{b}\left(\Delta_{1} * \Delta_{2}\right)=\sum_{v+w=b} f_{v}\left(\Delta_{1}\right) \cdot f_{w}\left(\Delta_{2}\right)$, we have $f\left(\Delta_{1} * \Delta_{2}, \mathbf{t}\right)=f\left(\Delta_{1}, \mathbf{t}\right) \cdot f\left(\Delta_{2}, \mathbf{t}\right)$. Then

$$
\begin{aligned}
h\left(\Delta_{1} * \Delta_{2}, \mathbf{t}\right) & =(1-\mathbf{t})^{a+a^{\prime}} f\left(\Delta_{1} * \Delta_{2}, \mathbf{t} / 1-\mathbf{t}\right) \\
& =(1-\mathbf{t})^{a} f\left(\Delta_{1}, \mathbf{t} / 1-\mathbf{t}\right) \cdot(1-\mathbf{t})^{a^{\prime}} f\left(\Delta_{2}, \mathbf{t} / 1-\mathbf{t}\right) \\
& =h\left(\Delta_{1}, \mathbf{t}\right) \cdot h\left(\Delta_{2}, \mathbf{t}\right) .
\end{aligned}
$$

Equating coefficients of these polynomials gives

$$
h_{b}\left(\Delta_{1} * \Delta_{2}\right)=\sum_{v+w=b} h_{v}\left(\Delta_{1}\right) \cdot h_{w}\left(\Delta_{2}\right)
$$

Corollary 4. If $\Delta_{1}, \ldots, \Delta_{n}$ are balanced complexes, then $\Delta_{1} * \cdots * \Delta_{n}$ is balanced, and $h_{b}\left(\Delta_{1} * \cdots * \Delta_{n}\right)=\sum_{v_{1}+\cdots+v_{n}=b} h_{v_{1}}\left(\Delta_{1}\right) \cdots \cdots h_{v_{n}}\left(\Delta_{n}\right)$, where $v_{i}$ is a vector, $1 \leq$ $\boldsymbol{i} \leq n$. 
Corollary 5. Let $\Delta_{1}, \ldots, \Delta_{n}$ be balanced complexes with disjoint labeling sets; say $\Delta_{i}$ is type $\mathbf{a}_{i}=\left(a_{i 1}, \ldots, a_{i m_{i}}\right)$ for $1 \leq i \leq n$. Then $\Delta_{1} * \cdots * \Delta_{n}$ is balanced of type $a=\left(\mathbf{a}_{1}, \ldots, \mathbf{a}_{n}\right)$ and for all $b \leq a, h_{b}\left(\Delta_{1} * \cdots * \Delta_{n}\right)=h_{b_{1}}\left(\Delta_{1}\right) \cdots \cdot h_{b_{n}}\left(\Delta_{n}\right)$, where $\mathbf{b}_{i}=\left(b_{i 1}, \ldots, b_{i m_{i}}\right)$.

Proposition 6. If $\hat{\Delta}$ is the minimal complex of type $a=\left(a_{1}, \ldots, a_{m}\right)$, then for all $b \leq a, h_{b}(\hat{\Delta})=1$.

Proof. Recall $\hat{\Delta}=\partial S_{1} * \cdots * \partial S_{m}$, where $S_{i}$ is an $a_{i}$-simplex, and the vertices in $S_{i}$ are labeled $i$. Let $b=\left(b_{1}, \ldots, b_{m}\right)$. Then $h_{b}(\hat{\Delta})=h_{b}\left(\partial S_{1} * \cdots * \partial S_{m}\right)=$ $h_{b_{1}}\left(\partial S_{1}\right) \cdots h_{b_{m}}\left(\partial S_{m}\right)$ by Corollary 5 . Since

$$
h\left(\partial S_{i}\right)=(\underbrace{1, \ldots, 1}_{a_{i}+1}, 0, \ldots, 0),
$$

and $b_{i} \leq a_{i}$ for all $i, h_{b_{i}}\left(\partial S_{i}\right)=1$ for $1 \leq i \leq m$. Thus $h_{b}(\hat{\Delta})=1$.

\section{A Balanced Stellar Subdivision}

One technique often used in generating new complexes from existing ones is stellar subdivision. In this section we introduce a balanced version of the concept, and examine how such an operation affects the extended $h$-vector.

Suppose $\Delta$ is a balanced simplicial complex of type $a=\left(a_{1}, \ldots, a_{m}\right)$, and $\sigma \in \Delta$ is a face of type $x \leq a$. Let $\hat{\Delta}$ denote the minimal type $x$ complex, and $\hat{\sigma}$ a maximal face in $\hat{\Delta}$. Then define the balanced stellar subdivision of $\sigma$ in $\Delta$ to be

$$
\operatorname{st}_{\text {bal }}(\Delta, \sigma) \equiv(\Delta \backslash \sigma) \cup\left[l k_{\Delta} \sigma *(\hat{\Delta} \backslash \hat{\sigma})\right],
$$

where $\sigma$ and $\hat{\sigma}$ are identified by a label-preserving bijection of their vertices.

\section{Examples}

(1) $a=(2,1), x=(1,1)$
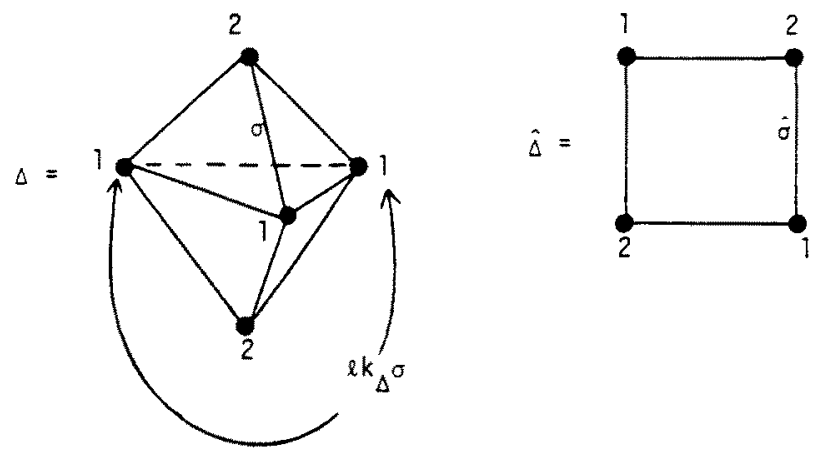


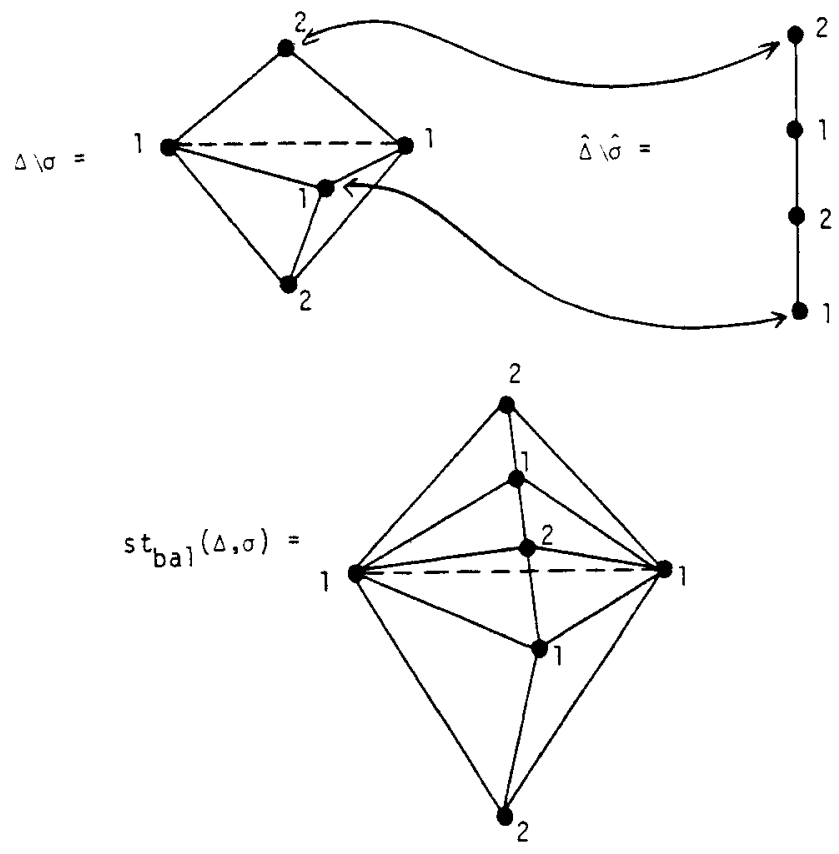

(2) $a=(1,1), x=(1,1)$
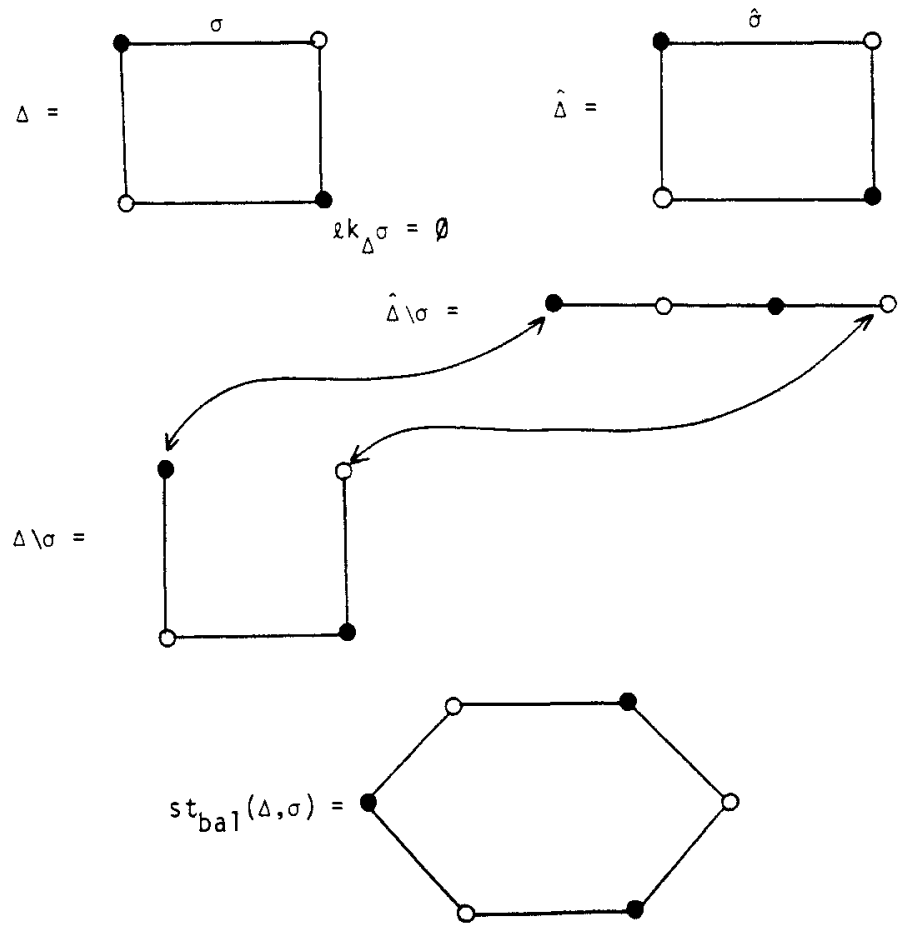
To show that $\operatorname{st}_{\mathrm{bal}}(\Delta, \sigma)$ is balanced of type $a$, we examine its maximal faces and see they are of two types:

(1) maximal faces of $\Delta$ not containing $\sigma$, and

(2) faces which are obtained by the union of a maximal face in $l k_{\Delta} \sigma$ with a maximal face in $\hat{\Delta} \backslash \hat{\sigma}$.

Faces of the first kind are clearly type $a$. Suppose $\tau$ is a face of the second kind, that is, $\tau=\gamma \cup \delta$ where $\gamma$ is a maximal face in $l k_{\Delta} \sigma$ and $\delta$ is maximal in $\hat{\Delta} \backslash \hat{\sigma}$. Then $\delta$ is type $x ; \gamma \cup \sigma$ is a face in $\Delta$, and is maximal since $\gamma$ is, so $\gamma \cup \sigma$ is type $a$. Since $\sigma$ is type $x, \gamma$ must be type $a-x$. Hence $\tau=\gamma \cup \delta$ is type $(a-x)+x=a$, showing $\operatorname{st}_{\mathrm{bal}}(\Delta, \sigma)$ is balanced of type $a$.

Another useful fact emerges from the foregoing discussion, which we record here for future reference.

Lemma 7. If $\Delta$ is a balanced complex of type $a$, and $\sigma \in \Delta$ is a face of type $x \leq a$, then $l k_{\Delta} \sigma$ is a balanced complex of type $a-x$.

We now consider how, for $\Delta$ minimal and $\sigma \in \Delta$, $\mathrm{st}_{\mathrm{bal}}(\Delta, \sigma)$ may be realized as the boundary complex of a simplicial polytope.

Theorem 8. Suppose $\Delta$ is the minimal type $a=\left(a_{1}, \ldots, a_{m}\right)$ complex, and $\sigma \in \Delta$ is a face of type $x$. Then there exists a simplicial d-polytope $P^{x}\left(d \equiv \sum_{i=1}^{m} a_{i}\right)$ and $a$ labeling of the vertices of $P^{x}$ such that $\partial P^{x}=\mathrm{st}_{\mathrm{bal}}(\Delta, \sigma)$.

Proof. We give here the basic idea of the proof, which generalizes that of Theorem 3.1 of [5]. See [15] for details in the general case.

Let $Q$ be the minimal polytope of type $a$; let $x=\left(x_{1}, \ldots, x_{m}\right)$. For $x=0$, the theorem is satisfied by letting $P^{x}$ be $Q$. So we may assume that $x_{1}, \ldots, x_{r}>0$, $x_{r+1}=\cdots=x_{m}=0$, for some $r>0$.

Starting with any face $F_{1}$ of type $x$, form the polytope $Q_{1}=\operatorname{st}_{Q}\left(F_{1}, t_{1}\right)$, where $t_{1}$ is a new vertex labeled 1 . Unless $r=1, Q_{1}$ is no longer balanced, as it has a face $F_{2}$ with $a_{1}+1$ vertices of label 1 . Form $Q_{2}=\operatorname{st}_{Q_{1}}\left(F_{2}, t_{2}\right)$ where $t_{2}$ has label 2. If $r>2$, then $Q_{2}$ will have a face $F_{3}$ with $a_{2}+1$ vertices of label 2. Continue until defining $Q^{r}$ which can be taken to be the polytope $P^{x}$.

\section{Example}

$a=(2,1), x=(1,1)$

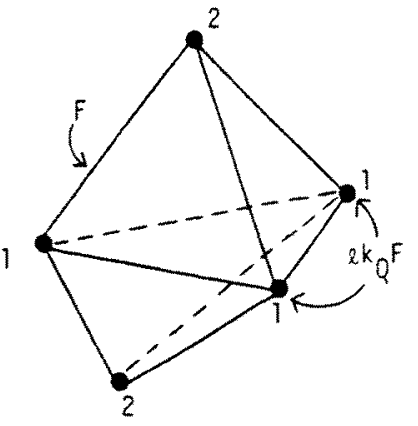

Q (solid)

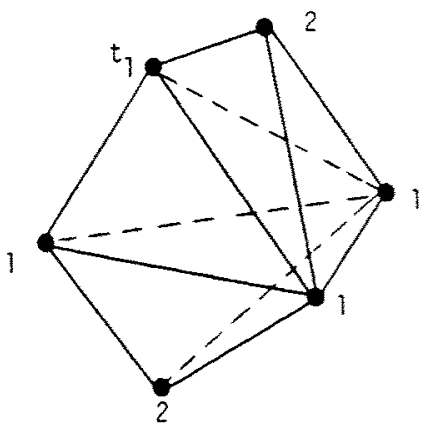

$Q_{1}$ 


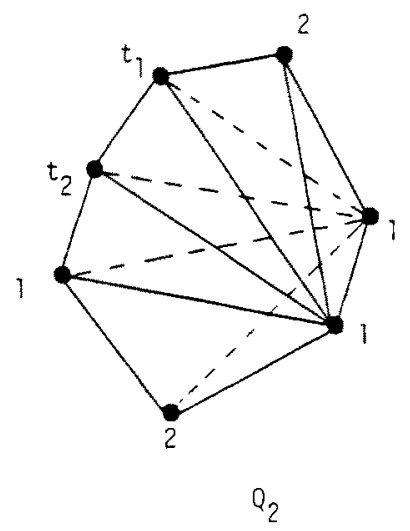

In fact, the construction in the proof shows that if $\Delta$ is any balanced simplicial type $a=\left(a_{1}, \ldots, a_{m}\right)$ complex and $\sigma \in \Delta$ is a type $x$ face involving $r$ labels, $1 \leq r \leq m$, then $\operatorname{st}_{\mathrm{bal}}(\Delta, \sigma)$ is obtained by a sequence of $r$ ordinary stellar subdivisions. A similar procedure can be followed to show that any pure labeled complex can be stellar subdivided into a type $a$ balanced complex (maintaining existing labels) for any vector $a$ not obviously excluded by the existing labels (i.e., the type allows for at least as many labels as have already been assigned). See Proposition 3.3. of [15].

There remains the computation of the extended $h$-vector of $\mathrm{st}_{\mathrm{bat}}(\Delta, \sigma)$.

Lemma 9. If $\Delta$ is the minimal type $a=\left(a_{1}, \ldots, a_{m}\right)$ complex, and $\sigma \in \Delta$ is a face of type $x \leq a$, then $l k_{\Delta} \sigma$ is the minimal type $a-x$ complex.

Proof. By Lemma $7, l k_{\Delta} \sigma$ is a balanced (simplicial) complex of type $a-x$. We first show that $f_{0}\left(l k_{\Delta} \sigma\right)=f_{0}\left(\Delta^{\prime}\right)$, where $\Delta^{\prime}$ is the minimal type $a-x$ complex. Remark 1 preceding Proposition 3 established that $f_{0}\left(\Delta^{\prime}\right)=$ $\sum_{i=1}^{m}\left(a_{i}-x_{i}+1\right)-\left|\left\{i: a_{i}=x_{i}\right\}\right|$. Without loss of generality, assume that $a_{i} \neq 0$ for each $i$, since otherwise $a_{i}=x_{i}=0$ and there would be no vertices labeled $i$ in either $\Delta$ or $\Delta^{\prime}$. Now, suppose $\sigma_{1}=\sigma_{1} * \sigma_{2} * \cdots * \sigma_{m}$, where $\sigma_{i}$ consists of all the vertices of $\sigma$ having label $i$. Then

$$
l k_{\Delta} \sigma=l k_{\partial S_{1}} \sigma_{1} * \cdots * l k_{\partial S_{m}} \sigma_{m}
$$

where the $S_{i}$ are as in Section 3. Thus

$$
f_{0}\left(l k_{\Delta} \sigma\right)=\sum_{i=1}^{m} f_{0}\left(l k_{\partial S_{i}} \sigma_{i}\right)
$$

If $a_{i}=x_{i}$ then $l k_{\partial S_{i}} \sigma_{i}=\varnothing$ and if $a_{i}>x_{i}$ then $f_{0}\left(l k_{\partial S_{i}} \sigma_{i}\right)=a_{i}+1-x_{i}$. So $f_{0}\left(l k_{\Delta} \sigma\right)=$ $f_{0}\left(\Delta^{\prime}\right)$ and thus $l k_{\Delta} \sigma$ is combinatorially equivalent to $\Delta^{\prime}$ by the uniqueness of $\Delta^{\prime}$. 
Theorem 10. If $\Delta$ is a minimal type $a=\left(a_{1}, \ldots, a_{m}\right)$ complex, and $\sigma \in \Delta$ is a face of type $x \leq a$, then for all $b \leq a$

$$
h_{b}\left(\mathrm{st}_{\mathrm{bal}}(\Delta, \sigma)\right)=1+\left|\left\{(y, z): y, z \in \mathbb{N}^{m}, y+z=b, 0 \leq y \leq a-x, 0 \varsubsetneqq z \leqq x\right\}\right| \text {. }
$$

Proof. First, if $\Delta_{1}$ and $\Delta_{2}$ are balanced complexes of type $a$, then $f\left(\Delta_{1} \cup \Delta_{2}, t\right)=$ $f\left(\Delta_{1}, \mathbf{t}\right)+f\left(\Delta_{2}, \mathbf{t}\right)-f\left(\Delta_{1} \cap \Delta_{2}, \mathbf{t}\right)$. So

$$
\begin{aligned}
h\left(\Delta_{1} \cup \Delta_{2}, \mathbf{t}\right)= & (1-\mathbf{t})^{a} f\left(\Delta_{1} \cup \Delta_{2}, \mathbf{t} / 1-\mathbf{t}\right) \\
= & (1-\mathbf{t})^{a} f\left(\Delta_{1}, \mathbf{t} / 1-\mathbf{t}\right)+(1-\mathbf{t})^{a} f\left(\Delta_{2}, \mathbf{t} / 1-\mathbf{t}\right) \\
& -(1-\mathbf{t})^{a} f\left(\Delta_{1} \cap \Delta_{2}, \mathbf{t} / 1-\mathbf{t}\right) \\
= & h\left(\Delta_{1}, \mathbf{t}\right)+h\left(\Delta_{2}, \mathbf{t}\right)-h^{(a)}\left(\Delta_{1} \cap \Delta_{2}, \mathbf{t}\right) .
\end{aligned}
$$

Now apply this to $\mathrm{st}_{\mathrm{bal}}(\Delta, \sigma) \equiv \Delta_{1} \cup \Delta_{2}$, where $\Delta_{1}=\Delta \backslash \sigma$, and $\Delta_{2}=l k_{\Delta} \sigma *(\hat{\Delta} \backslash \hat{\sigma})$. Recall that $\sigma$ and $\hat{\sigma}$ are identified by a bijection between vertex sets which preserves labeling; thus $\Delta_{1} \cap \Delta_{2}=(\Delta \backslash \sigma) \cap\left[l k_{\Delta} \sigma *(\hat{\Delta} \backslash \hat{\sigma})\right]=l k_{\Delta} \sigma * \partial \bar{\sigma}$. Note also that the complexes $\Delta_{1}$ and $\Delta_{2}$ are type a. So $h\left(\mathrm{st}_{\mathrm{bal}}(\Delta, \sigma), \mathrm{t}\right)=$ $h(\Delta \backslash \sigma, \mathbf{t})+h\left(l k_{\Delta} \sigma *(\hat{\Delta} \backslash \hat{\sigma}), \mathbf{t}\right)-h^{(a)}\left(l k_{\Delta} \sigma * \partial \bar{\sigma}, \mathbf{t}\right)$. Since $\Delta$ is the union of the type $a$ complexes $\Delta \backslash \sigma$ and $\bar{\sigma} * l k_{\Delta} \sigma$, and $(\Delta \backslash \sigma) \cap\left(\bar{\sigma} * l k_{\Delta} \sigma\right)=l k_{\Delta} \sigma * \partial \bar{\sigma}$, we also have $h(\Delta, \mathbf{t})=h(\Delta \backslash \sigma, \mathbf{t})+h\left(\bar{\sigma} * l k_{\Delta} \sigma, \mathbf{t}\right)-h^{(a)}\left(l k_{\Delta} \sigma * \partial \bar{\sigma}, \mathbf{t}\right)$. Combining these two equations gives

$$
\begin{array}{r}
h\left(\mathrm{st}_{\mathrm{bal}}(\Delta, \sigma), \mathbf{t}\right)=h(\Delta, \mathbf{t})-h\left(\bar{\sigma} * l k_{\Delta} \sigma, \mathbf{t}\right)+h^{(a)}\left(l k_{\Delta} \sigma * \partial \bar{\sigma}, \mathbf{t}\right) \\
+h\left(l k_{\Delta} \sigma *(\hat{\Delta} \backslash \hat{\sigma}), \mathbf{t}\right)-h^{(a)}\left(l k_{\Delta} \sigma * \partial \bar{\sigma}, \mathbf{t}\right) \\
=h(\Delta, \mathbf{t})+h\left(l k_{\Delta} \sigma, \mathbf{t}\right)[h(\hat{\Delta} \backslash \hat{\sigma}, \mathbf{t})-h(\bar{\sigma}, \mathbf{t})],
\end{array}
$$

the last equality using the fact that the $h$-polynomial of a join is the product of the $h$-polynomials of the complexes joined, established in the proof of Proposition 3.

By shelling the single simplex $\bar{\sigma}$ and appealing to Proposition 3.6 of [21], we see that $h(\bar{\sigma}, \mathbf{t})=1$. Similarly, shell $\hat{\Delta}$ so that $\hat{\sigma}$ is the last simplex in the shelling order. (See [7].) Since $\hat{\Delta}$ is minimal, Proposition 6 (this paper) and Proposition 3.6 of [21] yield

$$
h_{b}(\hat{\Delta} \backslash \hat{\sigma})= \begin{cases}1 & \text { for all } b \leq x, \\ 0 & \text { else. }\end{cases}
$$

So

$$
h_{b}(\hat{\Delta} \backslash \hat{\sigma})-h_{b}(\tilde{\sigma})= \begin{cases}1 & \text { for all } b<x, b \neq 0, \\ 0 & \text { else. }\end{cases}
$$

Recalling that $\Delta$ is minimal of type $a$, and $l k_{\Delta} \sigma$ is minimal of type $a-x$ gives

$$
h_{b}(\Delta)= \begin{cases}1 & \text { for all } b \leq a, \\ 0 & \text { else }\end{cases}
$$


and

$$
h_{b}\left(l k_{\Delta} \sigma\right)= \begin{cases}1 & \text { for all } b \leq a-x \\ 0 & \text { else }\end{cases}
$$

Hence for fixed $b \leq a$ the equation of $h$-polynomials yields $h_{b}\left(\mathrm{st}_{\mathrm{bal}}(\Delta, \sigma)\right)=$ $1+$ (the number of ways $b$ may be written as $y+z$ with $0 \leq y \leq a-x$ and $0 \leqq z \leqq$ $x)=1+\left|\left\{(y, z): y, z \in \mathbb{N}^{m}, y+z=b, 0 \leq y \leq a-x, 0 \leqq z \leqq x\right\}\right|$.

\section{An Affine Spanning Set for Balanced Spheres}

We now turn our attention back to the problem of determining the dimension of aff $\left(h_{b}\left(C_{a}^{d}\right)\right)$. For convenience, the notation is reviewed here. $C_{a}^{d}$ denotes the set of all type $a=\left(a_{1}, \ldots, a_{m}\right)$ balanced homology $(d-1)$-spheres and $\left(h_{b}\left(C_{a}^{d}\right)\right)$ the set of all vectors $\left(h_{b}(\Delta)\right)_{b \leq a} \in \mathbb{N}^{K}$ for $\Delta \in C_{a}^{d}$, where $K=\prod_{i=1}^{m}\left(a_{i}+1\right)$.

It was shown in Corollary 2 that $\operatorname{dim} \operatorname{aff}\left(h_{b}\left(C_{a}^{d}\right)\right) \leq\lfloor(K-1) / 2\rfloor$, and the aim here is to demonstrate equality. This is accomplished by obtaining $\lfloor(K-1) / 2\rfloor+1$ affinely independent vectors in $\operatorname{aff}\left(h_{b}\left(C_{a}^{d}\right)\right)$. At the same time, by using ideas developed in the two previous sections, we exhibit a set of balanced simplicial polytopes whose extended $h$-vectors span aff $\left(h_{b}\left(C_{a}^{d}\right)\right)$.

For $x \leq a$ let $P^{x}$ be the minimal type $a$ complex subdivided over a face of type $x$. Define a lexicographic order on all $x \leq a$ as follows: $x<y$ if $x_{i}<y_{i}$ for some $i, 1 \leq i \leq m$ and $x_{j}=y_{j}$ for all $j<i$. Define a $K \times K$ matrix $M$ whose columns are indexed by $b \leq a$, written in this lexicographic order, and whose rows are indexed by $x \leq a$, same order. The $(x, b)$-entry of $M$ is

$$
h_{b}\left(P^{x}\right)=1+\left|\left\{(y, z): y, z \in \mathbb{N}^{m}, y+z=b, 0 \leq y \leq a-x, 0 \leqq z ફ x\right\}\right| .
$$

We will show that $M$ has rank $\lfloor(K-1) / 2\rfloor+1$.

First, note that $h_{b}\left(P^{0}\right)=1$ for all $b$, since $x=0$ precludes the possibility of any $z$. So subtract this row from all others to get the matrix $M^{\prime}$ whose $(x, b)$-entry is $h_{b}\left(P^{x}\right)-1$ for all $x \leq a, x \neq 0$, and whose 0 row is all 1 's; this operation does not change rank.

Next, consider the related matrix $M^{\prime \prime}-M_{1}-M_{2}$, all matrices indexed by row and column as before, where $M^{\prime \prime}$ is an adjustment of $M^{\prime}$ which allows $z=0$ and $z=x, M_{1}$ corrects for the adjustment $z=0$, and $M_{2}$ for $z=x$. Then the $(x, b)$-entry of $M^{\prime \prime}$ is $\left|\left\{(y, z): y, z \in \mathbb{N}^{m}, y+z=b, 0 \leq y \leq a-x, 0 \leq z \leq x\right\}\right|$, the $(x, b)$-entry of $M_{1}$ is 1 if $0 \leq b \leq a-x, 0$ else, and the $(x, b)$-entry of $M_{2}$ is 1 if $x \leq b \leq a, 0$ else. $M^{\prime}$ differs from $M^{\prime \prime}-M_{1}-M_{2}$ only in the 0 row, which in $M^{\prime \prime}-M_{1}-M_{2}$ is all -1 's, so rank is the same.

Further examination of the matrices $M^{\prime \prime}, M_{1}$, and $M_{2}$ reveals that each has an underlying structure which turns out to be the key to the determination of the rank of $M$. This structure is uncovered in the following series of lemmas. 
Lemma 11. Let $a \geq 0$ be an integer. Then for integers $0 \leq x \leq a / 2$ and $0 \leq b \leq a$, the function $N(x, b) \equiv\{\{y, z): y, z \in \mathbb{Z}, y+z=b, 0 \leq y \leq a-x, 0 \leq z \leq x\} \mid$ takes on values

$$
N(x, b)= \begin{cases}b+1 & \text { if } 0 \leq b \leq x \\ x+1 & \text { if } x+1 \leq b \leq a-x \\ a-b+1 & \text { if } a-x+1 \leq b \leq a\end{cases}
$$

Proof. Set up a matrix of size $(a-x+1) \times(x+1)$, where the rows are indexed by $y=0,1,2, \ldots, a-x$, and the columns by $z=0,1,2, \ldots, x$. Note that since $x \leq a / 2$ there are at least as many rows as columns. Define the $(y, z)$-entry to be $y+z$. Then the entries on a given reverse diagonal are all the same, and if $b$ is the entry, $N(x, b)$ is the number of entries on that diagonal. So, if $0 \leq b \leq x$, the entries on the " $b$ " diagonal are $b+0,(b-1)+1, \ldots, 1+(b-1), 0+b$, amounting to $b+1$ entries in all. Similarly, looking at the lower right corner of the matrix, if $a-x+1 \leq b \leq a$, the entries on the " $b$ " diagonal are $x+(b-x),(x-1)+$ $(b-x+1), \ldots,(x-(a-b))+(a-x)$, totaling $a-b+1$ entries. In between, that is if $x+1 \leq b \leq a-x$, the number of entries on the " $b$ " diagonal remains constant at $x+1$. Specifically the entries are $b+0,(b-1)+1, \ldots,(b-x)+x$.

\section{Example}

$a=7, x=2$

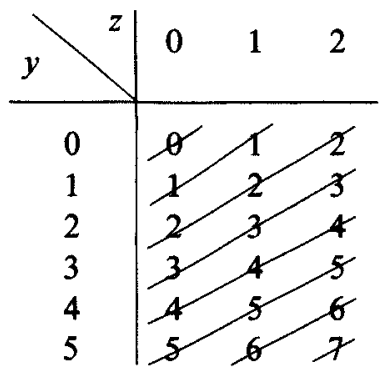

\begin{tabular}{|c|c|c|}
\hline$b$ & \multicolumn{2}{|c|}{$N(x, b)$} \\
\hline 0 & $1)$ & \\
\hline 1 & $2\}$ & $b+1$ \\
\hline 2 & 3 & \\
\hline 3 & 3) & \\
\hline 4 & $3\}$ & $x+1$ \\
\hline 5 & $3)$ & \\
\hline 6 & $2\}$ & $a-h+1$ \\
\hline 7 & $1\}$ & $a-b+1$ \\
\hline
\end{tabular}

Next let $n_{i}=a_{i}+1,1 \leq i \leq m$. We define $n_{i} \times n_{i}$ matrices $A_{i}$, for $i=1, \ldots, m$. Index the rows and columns of $A_{i}$ by $x_{i}=0,1, \ldots, a_{i}$, and $b_{i}=0,1, \ldots, a_{i}$, respectively, and let the $\left(x_{i}, b_{i}\right)$-entry be

$$
N\left(x_{i}, b_{i}\right) \equiv\left|\left\{(y, z): y, z \in \mathbb{Z}, y+z=b_{i}, 0 \leq y \leq a_{i}-x_{i}, 0 \leq z \leq x_{i}\right\}\right| .
$$

Despite its complicated definition, $A_{i}$ has a remarkably simple form.

\section{Examples}

(1) For $a_{i}=4, n_{i}=5$

$$
A_{i}=\left[\begin{array}{lllll}
1 & 1 & 1 & 1 & 1 \\
1 & 2 & 2 & 2 & 1 \\
1 & 2 & 3 & 2 & 1 \\
1 & 2 & 2 & 2 & 1 \\
1 & 1 & 1 & 1 & 1
\end{array}\right]
$$


(2) For $a_{i}=5, n_{i}=6$

$$
A_{i}=\left[\begin{array}{llllll}
1 & 1 & 1 & 1 & 1 & 1 \\
1 & 2 & 2 & 2 & 2 & 1 \\
1 & 2 & 3 & 3 & 2 & 1 \\
1 & 2 & 3 & 3 & 2 & 1 \\
1 & 2 & 2 & 2 & 2 & 1 \\
1 & 1 & 1 & 1 & 1 & 1
\end{array}\right]
$$

Lemma 12. $A_{i}$ has the following symmetries:

$$
N\left(x_{i}, b_{i}\right)=N\left(a_{i}-x_{i}, b_{i}\right)=N\left(x_{i}, a_{i}-b_{i}\right) .
$$

In addition, row $x_{i}$ of the $\left(\left\lfloor a_{i} / 2\right\rfloor+1\right) \times\left(\left\lfloor a_{i} / 2\right\rfloor+1\right)$ submatrix in the upper left corner of $A_{i}$ is

$$
\begin{array}{llllllll}
1 & 2 & \cdots & x_{i} & x_{i}+1 & x_{i}+1 & \cdots & x_{i}+1 .
\end{array}
$$

Proof. The proof is a routine verification using Lemma 11.

Let $A_{1} \otimes A_{2}$ denote the tensor product of the matrices $A_{1}$ and $A_{2}$. That is, $A_{1} \otimes A_{2}$ is the $n_{1} n_{2} \times n_{1} n_{2}$ matrix consisting of rows (and columns) of $n_{1}$ square blocks of size $n_{2}$; the block in the $k$ th row and $l$ th column is $A_{2}$ multiplied by the scalar in the $k$ th row and $l$ th column of $A_{1}, 1 \leq k, l \leq n_{1}$. This product is associative, so the iterated tensor product of $A_{1}, \ldots, A_{m}$ is denoted $A_{1} \otimes \cdots \otimes A_{m}$ (without parentheses), alternatively $\otimes_{i=1}^{m} A_{i}$. The rows and columns of $\bigotimes_{i=1}^{m} A_{i}$ are indexed by $x \leq a$ and $b \leq a$, respectively, written in the lexicographic order defined earlier. So the $\left(\left(x_{1}, x_{2}, \ldots, x_{m}\right),\left(b_{1}, b_{2}, \ldots, b_{m}\right)\right)$-entry of $\otimes_{i=1}^{m} A_{i}$ is given by $\prod_{i=1}^{m}\left[\left(x_{i}, b_{i}\right)\right.$-entry of $\left.A_{i}\right]$. For the basic facts about tensor products used here, refer to [14].

Now denote by $I_{n_{i}}$ the $n_{i} \times n_{i}$ identity matrix, and by $J_{n_{1}}$ the matrix with the columns of $I_{n_{i}}$ written in reverse order. Let $E_{i}$ be the $n_{i} \times n_{i}$ upper triangular matrix of 1's, and let $C_{i}=E_{i} I_{n_{i}}$, so $C_{i}$ has the columns of $E_{i}$ written in reverse order:

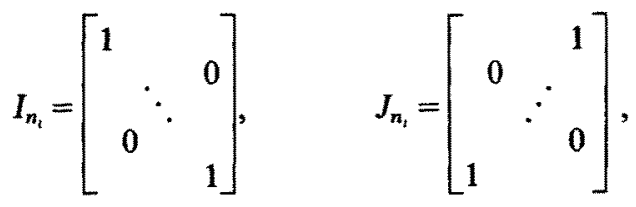

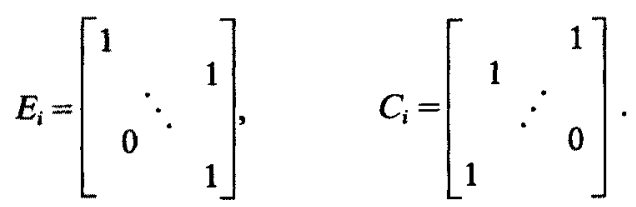

Lemma 13. In the setting described above,

(i) $M^{\prime \prime}=\bigotimes_{i=1}^{m} A_{i}$,

(ii) $M_{1}=\bigotimes_{i=1}^{m} C_{i}$,

(iii) $M_{2}=\bigotimes_{i=1}^{m} E_{i}$. 
Proof. (i) The $(x, b)$-entry of $M^{\prime \prime}$ is

$$
\begin{aligned}
\mid\left\{(y, z): y, z \in \mathbb{N}^{m}, y+z\right. & =b, 0 \leq y \leq a-x, 0 \leq z \leq x\} \mid \\
& =\prod_{i=1}^{m} N\left(x_{i}, b_{i}\right)=\prod_{i=1}^{m}\left[\left(x_{i}, b_{i}\right) \text {-entry of } A_{i}\right] \\
& =(x, b) \text {-entry of } \bigotimes_{i=1}^{m} A_{i} .
\end{aligned}
$$

(ii) The $\left(x_{i}, b_{i}\right)$-entry of $C_{i}$ is

$$
\begin{cases}1 & \text { if } x_{i}+b_{i} \leq a_{i} \\ 0 & \text { else }\end{cases}
$$

so the $(x, b)$-entry of $\otimes_{i=1}^{m} C_{i}$ is

$$
\begin{cases}1 & \text { if } x_{i}+b_{i} \leq a_{i} \text { for all } 1 \leq i \leq m \\ 0 & \text { else. }\end{cases}
$$

But the $(x, b)$-entry of $M_{3}$ is

$$
\left\{\begin{array}{ll}
1 & \text { if } 0 \leq b \leq a-x \\
0 & \text { else }
\end{array}=\left\{\begin{array}{ll}
1 & \text { if } x+b \leq a \\
0 & \text { else }
\end{array}=(x, b) \text {-entry of } \otimes_{i=1}^{m} C_{i} .\right.\right.
$$

(iii) The $\left(x_{i}, b_{i}\right)$-entry of $E_{i}$ is

$$
\begin{cases}1 & \text { if } b_{i} \geq x_{i} \\ 0 & \text { else, }\end{cases}
$$

so the $(x, b)$-entry of $\otimes_{i=1}^{m} E_{i}$ is

$$
\begin{cases}1 & \text { if } b_{i} \geq x_{i} \text { for all } 1 \leq i \leq m, \\ 0 & \text { else. }\end{cases}
$$

Finally, the $(x, b)$-entry of $M_{2}$ is

$$
\left\{\begin{array}{ll}
1 & \text { if } x \leq b \leq a \\
0 & \text { else. }
\end{array}=(x, b) \text {-entry of } \otimes_{i=1}^{m} E_{i} .\right.
$$

Hence the problem of establishing equality in Corollary 2 is reduced to that of showing the matrix $\left(\otimes_{i=1}^{m} A_{i}\right)-\left(\otimes_{i=1}^{m} C_{i}\right)-\left(\otimes_{i=1}^{m} E_{i}\right)$ to have rank $\geq\lfloor(K-$ $1) / 2\rfloor+1=\lceil K / 2\rceil$, where $K=\prod_{i=1}^{m}\left(a_{i}+1\right)=\prod_{i=1}^{m} n_{i}$.

Theorem 14. $\left(\otimes_{i=1}^{m} A_{i}\right)-\left(\otimes_{i=1}^{m} C_{i}\right)-\left(\bigotimes_{i=1}^{m} E_{i}\right)$ has rank $\geq\lceil K / 2\rceil$.

Proof:

$$
\begin{aligned}
\operatorname{rank}\left[\bigotimes_{i=1}^{m}\right. & \left.A_{i}-\bigotimes_{i=1}^{m} C_{i}-\bigotimes_{i=1}^{m} E_{i}\right] \\
& =\operatorname{rank}\left[\left(\bigotimes_{i=1}^{m} E_{i}\right)^{-1}\left(\bigotimes_{i=1}^{m} A_{i}-\bigotimes_{i=1}^{m} C_{i}-\bigotimes_{i=1}^{m} E_{i}\right)\right] \\
& =\operatorname{rank}\left[\left(\otimes_{i=1}^{m} E_{i}^{-1}\right)\left(\otimes_{i=1}^{m} A_{i}\right)-\left(\bigotimes_{i=1}^{m} E_{i}^{-1}\right)\left(\bigotimes_{i=1}^{m} C_{i}\right)-I_{K}\right] \\
& =\operatorname{rank}\left[\bigotimes_{i=1}^{m}\left(E_{i}^{-1} A_{i}\right)-\bigotimes_{i=1}^{m}\left(E_{i}^{-1} C_{i}\right)-I_{K}\right] \\
& =\operatorname{rank}\left[\bigotimes_{i=1}^{m}\left(E_{i}^{-1} A_{i}\right)-J_{K}-I_{K}\right]
\end{aligned}
$$


since

$$
\bigotimes_{i=1}^{m} E_{i}^{-1} C_{i}=\bigotimes_{i=1}^{m} J_{n_{i}} C_{i}^{-1} C_{i}=\bigotimes_{i=1}^{m} J_{n_{i}}=J_{\prod_{i=1}^{m} n_{i}}=J_{K}
$$

If $K$ is even, $-J_{K}-I_{K}$ is

$$
\left[\begin{array}{ccccccc}
-1 & & & & & & -1 \\
& \ddots & & 0 & & \cdot \cdot \\
& 0 & -1 & & -1 & & \\
& & -1 & & -1 & 0 & \\
-1 & & & & & \ddots & -1
\end{array}\right]_{K}
$$

and if $K$ is odd, $-J_{K}-I_{K}$ is

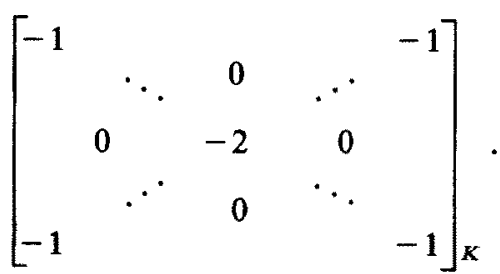

Whatever the case, denote this "correction" matrix by $T$.

The proof is completed by showing that the $[K / 2\rceil \times[K / 2]$ submatrix in the upper left corner of $\otimes_{i=1}^{m}\left(E_{i}^{-1} A_{i}\right)+T$ has rank $\lceil K / 2\rceil$. Recall that $E_{i}$ is upper triangular with 1 's, so $\left(E_{i}\right)^{-1}$ is obtained from $I_{n_{i}}$ by replacing row $j$ by [row $j-$ row $(j+1)$ ] for $1 \leq j \leq n_{i}-1$. Thus for each $1 \leq i \leq m, E_{i}^{-1} A_{i}$ has the following form: for $1 \leq j \leq\left\lceil\left(n_{i}-1\right) / 2\right\rceil$, row $j$ is $j 0$ 's, followed by $\left(n_{i}-2 j\right)-1$ 's, followed by $j 0$ 's; for $\left\lceil\left(n_{i}-1\right) / 2\right\rceil<j \leq n_{i}-1$, row $j$ is $-\left(\operatorname{row}\left(n_{i}-j\right)\right)$; row $n_{i}$ consists of 1 's.

\section{Examples}

(1) For $a_{i}=4, n_{i}=5$

$$
A_{i}=\left[\begin{array}{lllll}
1 & 1 & 1 & 1 & 1 \\
1 & 2 & 2 & 2 & 1 \\
1 & 2 & 3 & 2 & 1 \\
1 & 2 & 2 & 2 & 1 \\
1 & 1 & 1 & 1 & 1
\end{array}\right], \quad E_{i}^{-1} A_{i}=\left[\begin{array}{rrr:rr}
0 & -1 & -1 & -1 & 0 \\
0 & 0 & -1 & 0 & 0 \\
0 & 0 & 1 & 0 & 0 \\
\hdashline 0 & 1 & 1 & 1 & 0 \\
1 & 1 & 1 & 1 & 1
\end{array}\right] .
$$

(2) For $a_{i}=5, n_{i}=6$

$$
A_{i}=\left[\begin{array}{rrrrrr}
1 & 1 & 1 & 1 & 1 & 1 \\
1 & 2 & 2 & 2 & 2 & 1 \\
1 & 2 & 3 & 3 & 2 & 1 \\
1 & 2 & 3 & 3 & 2 & 1 \\
1 & 2 & 2 & 2 & 2 & 1 \\
1 & 1 & 1 & 1 & 1 & 1
\end{array}\right], \quad E_{i}^{-1} A_{i}=\left[\begin{array}{rrr:rrr}
0 & -1 & -1 & -1 & -1 & 0 \\
0 & 0 & -1 & -1 & 0 & 0 \\
0 & 0 & 0 & 0 & 0 & 0 \\
\hdashline 0 & 0 & 1 & 1 & 0 & 0 \\
0 & 1 & 1 & 1 & 1 & 0 \\
1 & 1 & 1 & 1 & 1 & 1
\end{array}\right] .
$$


So the $\left\lceil n_{i} / 2\right\rceil \times\left\lceil n_{i} / 2\right\rceil$ submatrix in the upper left corner of $E_{i}^{-1} A_{i}$ is

if $n_{i}$ is even, and

$$
\left[\begin{array}{rrr}
0 & & \\
& \ddots & -1 \\
& 0 & \\
& & 0
\end{array}\right]
$$

$$
\left[\begin{array}{cccc}
0 & & -1 & \\
& \ddots & & \\
& 0 & 0 & \\
& & & 1
\end{array}\right]
$$

if $n_{i}$ is odd.

Denote the $\lceil K / 2\rceil \times[K / 2\rceil$ upper left submatrix in $\otimes_{i=1}^{m} E_{i}^{-1} A_{i}$ by $S$.

Claim. $S$ is strictly upper triangular if $K$ is even, and of the form

$$
\left[\begin{array}{llll}
0 & & & \\
& \ddots & & \\
& 0 & 0 & \\
& & & 1
\end{array}\right]
$$

if $K$ is odd. Recall $K=\prod_{i=1}^{m} n_{i}$, and induct on $m$. If $m=1$, the assertion is true, as previously noted. So suppose for all $m<k$ the result holds and let $m=k$.

The induction hypothesis says that $\bigotimes_{i=2}^{k} E_{i}^{-1} A_{i}$ is strictly upper triangular if $\prod_{i=2}^{k} n_{i}$ is even, and of the form

$$
\left[\begin{array}{llll}
0 & & & \\
& \ddots & & \\
& 0 & 0 & \\
& & & 1
\end{array}\right]
$$

if $\prod_{i=2}^{k} n_{i}$ is odd. Also $E_{1}^{-1} A_{1}$ is strictly upper triangular if $n_{1}$ is even, and of the form

$$
\left[\begin{array}{llll}
0 & & & \\
& \ddots & & \\
& 0 & 0 & 1
\end{array}\right]
$$

if $n_{1}$ is odd.

Now suppose $K$ is even; then either $n_{1}$ is even, or $n_{1}$ is odd and $\prod_{i=2}^{k} n_{i}$ is even. In the first instance, $S$ has the form

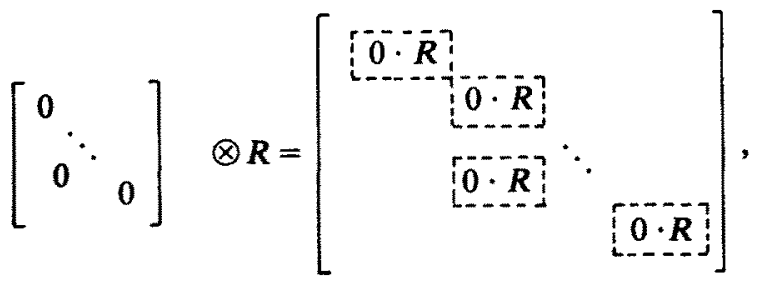


where $R$ denotes $\otimes_{i=2}^{k} E_{i}^{-1} A_{i}$, and the broken lines show the blocks on the diagonal and below the diagonal in $S$. In the second instance, $S$ has the form

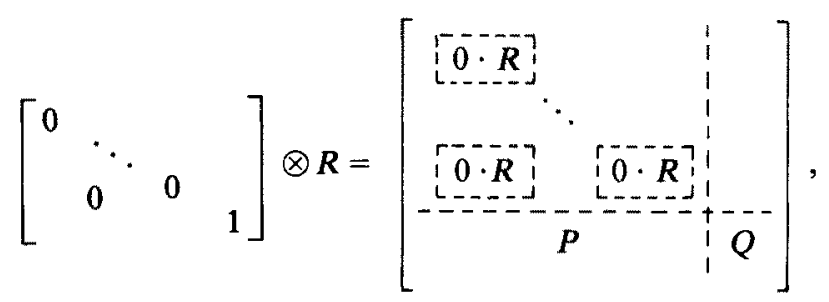

where the blocks in region $P$ consist of 0 . (the upper $\frac{1}{2}\left(\prod_{i=1}^{k} n_{i}\right.$ ) rows of $R$ ), hence $P$ is all 0 's; and $Q$ is 1 . (the upper left corner of $R$, size $\frac{1}{2}\left(\prod_{i=2}^{k} n_{i}\right)$ ). Since $\prod_{i=2}^{k} n_{i}$ is even, $Q$ is strictly upper triangular. Thus if $K$ is even, $S$ is strictly upper triangular.

On the other hand, if $K$ is odd, then $n_{1}$ must be odd and $\prod_{i=2}^{k} n_{i}$ must be odd. $S$ has the form

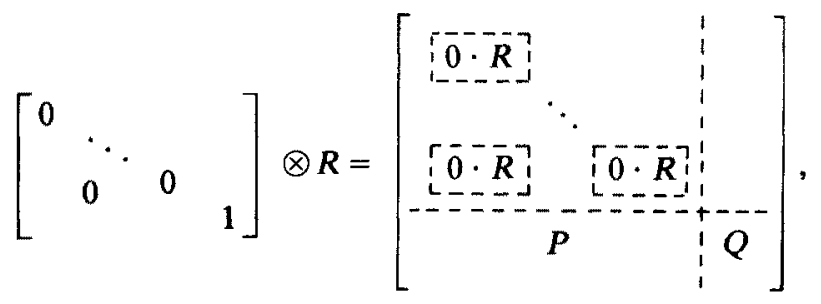

where the blocks in $P$ consist of $0 \cdot\left(\right.$ the upper $\frac{1}{2}\left(\prod_{i=2}^{k} n_{i}+1\right)$ rows of $R$ ), hence $P$ is all 0 's; and $Q$ is 1 . (the upper left corner of $R$, size $\frac{1}{2}\left(\prod_{i=2}^{k} n_{i}+1\right)$ ). Since $\prod_{i=2}^{k} n_{i}$ is odd, $Q$ has the form

$$
\left[\begin{array}{llll}
0 & & & \\
& \ddots & & \\
& 0 & 0 & \\
& & & 1
\end{array}\right]
$$

Thus for $K$ odd, $S$ has the form

$$
\left[\begin{array}{llll}
0 & & & \\
& \ddots & & \\
& 0 & 0 & \\
& & & 1
\end{array}\right],
$$

and this proves the claim. that

Finally, let $T^{\prime}$ be the upper left submatrix of the "correction" matrix $T$. Recall

$$
T^{\prime}=\left[\begin{array}{lllll}
-1 & & 0 & \\
& 0 & \ddots & & \\
& & & & -1
\end{array}\right]
$$


if $K$ is even, and

$$
\left[\begin{array}{rrrr}
-1 & & & \\
& \ddots & 0 & \\
& 0 & -1 & -2
\end{array}\right]
$$

if $K$ is odd. So if $K$ is even, $S+T^{\prime}$ has the form

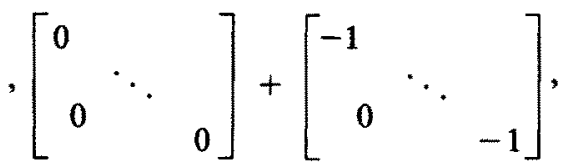

and if $K$ is odd, it has form

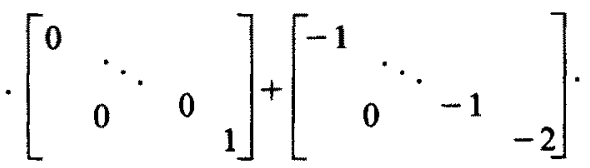

In either event $S+T^{\prime}$ is triangular; therefore its rank is $\lceil K / 2\rceil$.

\section{Some Habitats of Balanced Complexes}

Here we look at two settings in which balanced and completely balanced complexes arise naturally. One involves stellar subdivision of geometric cellular (not necessarily simplicial) complexes, so we offer a brief description of this based on discussions in Ewald and Shephard [9] and Bayer [3]. A few definitions are called for.

The cell $P$ is a $d$-pyramid if $P=\operatorname{conv}(B \cup\{v\})$ where $B$ (the base of $P$ ) is a $(d-1)$-cell and $v$ (the apex of $P$ ) is a point not contained in aff $B$. For $r$ a positive integer, $P$ is an $r$-fold $d$-pyramid with base $B$ of dimension $d-r$ if $P$ is a $d$-pyramid with base $P^{\prime}$ where $P^{\prime}$ is an $(r-1)$-fold $(d-1)$-pyramid with base $B$. Ewald and Shephard's generalization differs from the subdivision of simplicial polytopes mainly in its requirement that if the cell $F$ to be subdivided in a complex $P$ is contained in a cell $H$ of higher dimension, $H$ must be a $(\operatorname{dim} H-$ $\operatorname{dim} F$ )-fold pyramid with base $F$. The notion of link of $F$ in $P$ carries over intact so if a cell $F$ meets the criterion above, $l k_{P} F$ is simplicial. Since the definition of the join $*$ of simplicial complexes applies also to cellular complexes whose affine hulls are independent affine subspaces, $F$ may be subdivided as $F^{\prime} *\{t\} * G$ where $F^{\prime}$ is a proper cell of $F, G$ is a face in $l k_{P} F$, and $t$ is a point in the relative interior of $F$. The $d$-cells in a $d$-complex can always be subdivided, and the following fact from [9] sets the stage for subdivisions of lower-dimensional cells. Say $F$ is an $(r-1)$-cell in a complex $P$ for some $r, 1 \leq r \leq d-1$; if all $r$-cells in $P$ which contain $F$ have been subdivided, yielding a complex $P^{\prime}$, then all the cells of $P^{\prime}$ containing $F$ are pyramids with base $F$. Thus $F$ itself may be subdivided. So in some sense, the order of subdivision in a nonsimplicial complex proceeds by decreasing dimension.

We now go on to the examples. Grünbaum [11] defines a $d$-polytope to be $k$-simplicial if every $k$-face of $P$ is a simplex. When $k=d-2, P$ is also called 
quasi-simplicial; in this case the facets of $P$ are simplicial. For example, every 3-polytope is quasi-simplicial. If a stellar subdivision is performed on each facet of a quasi-simplicial $d$-polytope $P$, the resulting $d$-polytope $P^{\prime}$ is simplicial. This suggests a natural way to label the vertices of $P^{\prime}$ so that it is balanced of type $(d-1,1)$. Give the vertices of $P$ the label $A$, and assign each vertex introduced by a subdivision of a facet the label $B$. Then each facet of $P^{\prime}$ will have $d-1$ vertices labeled $A$, and 1 vertex labeled $B$.

\section{Example}
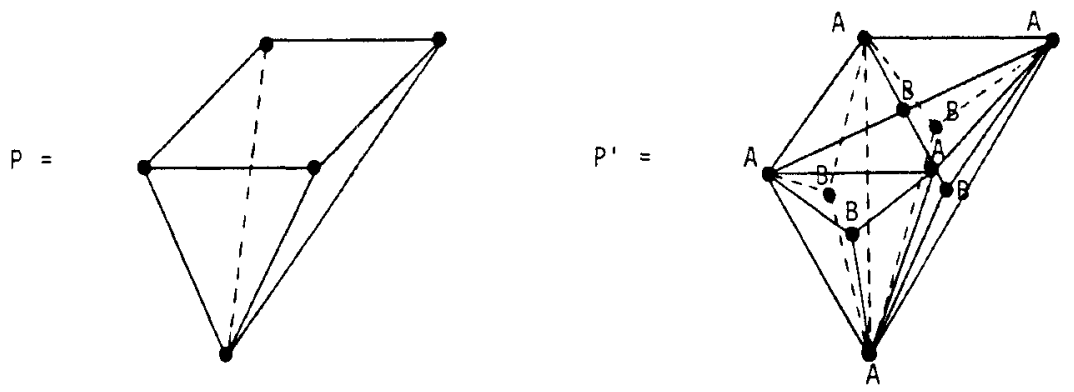

This idea generalizes to the $k$-simplicial case. If $P$ is $k$-simplicial, its nonsimplicial faces can be subdivided to yield a simplicial polytope $P^{\prime}$. Give the vertices of $P$ the label 0 . First subdivide all facets (simplices or not) using vertices labeled 1. Continue to subdivide all faces of a given dimension $d-i, 1 \leq i \leq d-k-1$, proceeding in order of decreasing dimension. The new vertices introduced in the subdivision of faces of dimension $d-i$ are labeled $i$. The polytope $P^{\prime}$ then has type $\left(a_{0}, a_{1}, \ldots, a_{d-k-1}\right)$ where $a_{0}=k+1$ and $a_{i}=1$ for $1 \leq i \leq d-k-1$. The label on each vertex conveniently keeps track of the stage in the subdivisions at which the vertex appeared in the polytope.

Example. Consider the 3-cube as a 1-simplicial facet of a 4-cube. The illustration shows the polytope obtained after the subdivision of the 3-face and two of the 2-faces. Note that the facets emerging are type $(2,1,1)$.

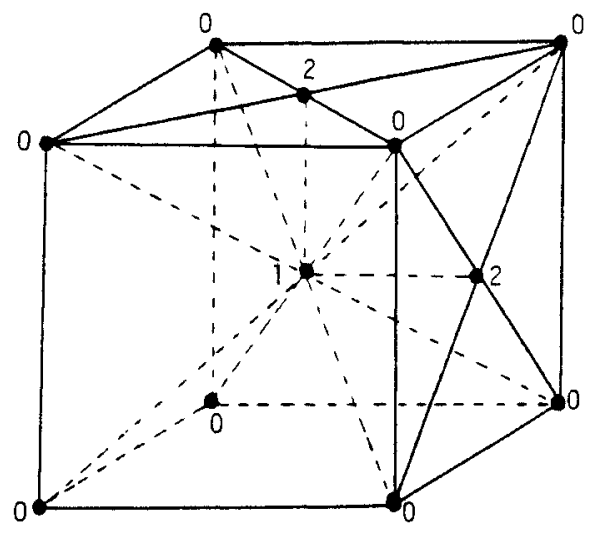


Since every $d$-polytope is $\mathbf{0}$-simplicial, if all faces of dimension 1 or more in $P$ are subdivided as described above, the resulting simplicial polytope is completely balanced. In this way we can achieve a simplicial polytope $P^{\prime}$ which is balanced of any type $a=\left(a_{1}, \ldots, a_{r}\right), r \leq d$, where $a_{i}$ is a positive integer and $\sum_{i=1}^{r} a_{i}=d$, just by identifying labels.

The question of whether certain complexes can be completely balanced may be couched in the language of graph colorability. A simplicial complex is $n$ colorable if each vertex can be labeled with some integer $i, 1 \leq i \leq n$, so that the vertices of any simplex have distinct labels. Thus if $\Delta$ is a pure simplicial $d$-complex, saying $\Delta$ is $(d+1)$-colorable is the same as saying $\Delta$ is completely balanced.

It is well known (see [2], for example) that a graph which can be embedded in the 2-sphere $S^{2}$ is 3-colorable if and only if it is a subcomplex of the 1-skeleton of a triangulation of $S^{2}$ in which every vertex has even degree. An analog of this result to higher dimensions was proved by Goodman and Onishi [10], and independently by Deligne, Edwards, MacPherson, and Morgan (announced in [8]). The general result asserts that a closed, simply connected triangulated $P L$ $n$-manifold is $(n+1)$-colorable if and only if each $(n-2)$-simplex is a face of an even number of $(n-1)$-simplices. (In [10], only the case $d=4$ is considered, but the proof applies to any dimension.)

\section{Acknowledgment}

We acknowledge many helpful discussions with Gil Kalai over the course of this work.

\section{References}

1. J. W. Alexander, The combinatorial theory of complexes, Ann. of Math. (2) 31 (1930), 292-320.

2. D. Barnette, Map Coloring, Polyhedra, and the Four-Color Problem, Dolciani Mathematical Expositions No. 8, Mathematical Association of America, Washington, DC, 1983.

3. M. M. Bayer, Facial Enumeration in Polytopes, Spheres and Other Complexes, Ph.D. thesis, Cornell University, Ithaca, NY, 1983.

4. M. M. Bayer and L. J. Billera, Counting faces and chains in polytopes and posets, Contemp. Math. 34 (1984), 207-252.

5. M. M. Bayer and L. J. Billera, Generalized Dehn-Sommerville relations for polytopes, spheres and Eulerian partially ordered sets, Invent. Math. 79 (1985), 143-157.

6. L. J. Billera, Polyhedral theory and commutative algebra, in Mathematical Programming -Bonn 1982, The State of the Art, (A. Bachem, M. Grötschel, and B. Korte, eds.), 57-77, Springer-Verlag, Berlin, 1983.

7. H. Bruggesser and P. Mani, Shellable decompositions of cells and spheres, Math. Scand. 29 (1971), 197-205.

8. R. D. Edwards, An amusing reformulation of the four-color problem, Notices Amer. Math. Soc. 24 (1977), A-257.

9. G. Ewald and G. C. Shephard, Stellar subdivisions of boundary complexes of convex polytopes, Math. Ann. 210 (1974), 7-16. 
10. J. E. Goodman and H. Onishi, Even triangulations of $S^{3}$ and the coloring of graphs, Trans. Amer. Math. Soc. 246 (1978), 501-510.

11. B. Grübaum, Convex Polytopes, Wiley-Interscience, New York, 1967.

12. D. W. Henderson, Simplicial complexes homeomorphic to proper self-subsets have free faces, in Continua, Decompositions, Manifolds, 240 242, University of Texas Press, Austin, TX, 1983.

13. V. Klee, A combinatorial analogue of Poincaré's duality theorem, Canad. J. Math. 16 (1964), 517-531.

14. C. C. MacDuffee, The Theory of Matrices, Verlag von Julius Springer, Berlin, 1933.

15. K. E. Magurn, Subdivision and Enumeration in Balanced Complexes, Ph.D. thesis, Cornell University, 1985.

16. W. S. Massey, Singular Homology Theory, Springer-Verlag, New York, 1980.

17. C. R. F. Maunder, Algebraic Topology, Van Nostrand Reinhold, London, 1970.

18. P. McMullen and G. C. Shephard, Convex Polytopes and the Upper Bound Conjecture, Cambridge University Press, Cambridge, 1971.

19. D. M. Y. Sommerville, The relations connecting the angle-sums and volume of a polytope in space of $n$ dimensions, Proc. Roy. Soc. London Ser. A 115 (1927), 103-119.

20. R. Stanley, Cohen-Macaulay complexes in Higher Combinatorics (M. Aigner, ed.), 51-62, Reidel, Dordrecht and Boston, 1977.

21. R. Stanley, Balanced Cohen-Macaulay complexes, Trans. Amer. Math. Soc. 249 (1979), 139-157.

22. R. Stanley, An introduction to combinatorial commutative algebra, in Enumeration and Design (D. A. Jackson and S. A. Vanstone, eds.), 3-18, Academic Press Canada, Toronto, 1984.

Received September 4, 1985, and in revised form August 14, 1986. 